\title{
Enhanced Matrix Metalloproteinase Activity in the Spontaneously Hypertensive Rat: VEGFR-2 Cleavage, Endothelial Apoptosis, and Capillary Rarefaction
}

\author{
Edward D. Tran Frank A. DeLano Geert W. Schmid-Schönbein \\ Department of Bioengineering, Whitaker Institute for Biomedical Engineering, University of California San Diego, \\ La Jolla, Calif., USA
}

\section{Key Words}

Capillary density $\cdot$ Hypertension $\cdot$ Matrix

metalloproteinases $\cdot$ Vascular endothelial growth factor

\begin{abstract}
Besides an elevated blood pressure, the spontaneously hypertensive rat (SHR) has multiple microvascular complications including endothelial apoptosis with capillary rarefaction. The SHR also has elevated levels of proteolytic (e.g. matrix metalloproteinase, MMP) activity and apoptosis in microvascular cells compared to its normotensive control, but the specific enzymes involved and the molecular mechanism for apoptosis are unknown. We hypothesize that selected MMPs cleave the extracellular domain of vascular endothelial growth factor receptor-2 (VEGFR-2), which in turn causes endothelial apoptosis and capillary rarefaction. Zymographic analysis shows that gelatinase (MMP-2 and MMP-9) and matrilysin (MMP-7) activities are significantly enhanced in SHR plasma. The SHR has lower levels of the extracellular domains of VEGFR-2 in cardiac microvessels. Furthermore, application of plasma from the $\mathrm{SHR}$, or purified MMP-9 and MMP-7 to naïve cells causes cleavage of the extracellular domain of VEGFR-2. The receptor cleavage was blocked by broad-acting MMP inhibitors (GM6001 $1 \mu \mathrm{M}$, EDTA $10 \mathrm{~mm}$, or doxycycline $11.3 \mu \mathrm{M})$. Chronic MMP inhibi-
\end{abstract}

tion (doxycycline, $5.4 \mathrm{mg} / \mathrm{kg} / \mathrm{day}, 24$ weeks) attenuated VEGFR-2 cleavage, endothelial apoptosis, and capillary rarefaction in the SHR. These results suggest elevated plasma MMP activities may cleave VEGFR-2, resulting in endothelial apoptosis and capillary rarefaction in the SHR.

Copyright $\odot 2010$ S. Karger AG, Basel

\section{Introduction}

Hypertension is associated with a high prevalence and an increased risk of cardiovascular morbidity and mortality. Besides an elevated arterial blood pressure, the spontaneously hypertensive rat (SHR) form of hypertension shows numerous microvascular complications, e.g. free radical production, insulin resistance, attenuated leukocyte adhesion to the endothelium, apoptosis, and enhanced organ injury [1-5]. Endothelial apoptosis causes capillary closure and rarefaction with loss of parenchymal cells [6].

The vascular endothelial growth factor receptor-2 (VEGFR-2) plays a central role in the proliferation and

Sources of support: NHLBI HL 10881 and a gift from Leading Ventures.

\section{KARGER}

두 2010 S. Karger AG, Basel

Fax +41613061234

E-Mail karger@karger.ch

www.karger.com www.karger.com/jvr
Dr. Edward D. Tran

Department of Bioengineering

Whitaker Institute for Biomedical Engineering

University of California San Diego, La Jolla, CA 92093-0412 (USA)

Tel. +1 858534 2714, Fax +1 858534 5722,E-Mail etran@ucsd.edu 
apoptosis of vascular endothelium [7-9]. Depletion of VEGFR-2 signaling leads to apoptosis in capillaries [10], but no molecular mechanism has been proposed for the enhanced endothelial apoptosis in hypertension.

The matrix metalloproteinase (MMP) family includes 24 members that share structural domains, but differ in substrate specificity, cellular sources, and transcriptional regulation [11]. Based on structural domains, the MMP family consists of five subgroups: collagenase, gelatinase, stromelysin, matrilysin, and membrane-type MMPs. MMPs degrade components of the extracellular matrix, remodel the extracellular matrix, and influence angiogenesis [12]. This feature is relevant for many aspects of mammalian biology and pathophysiology $[13,14]$.

Recently, we showed that SHRs have elevated levels of unchecked proteolytic activity in plasma and on endothelium compared to their normotensive control, the Wistar Kyoto (WKY) rat. MMPs and other enzymes contribute to this process [15]. MMPs cause cleavage of membrane receptors, e.g. the extracellular domain of the insulin receptor and consequently insulin resistance, or cleavage of CD18 on leukocytes and defective adhesion of leukocytes to the endothelium. Blockade of the MMPs prevents receptor cleavage and symptoms that are associated with insulin resistance and attenuated leukocyte adhesion, in addition to reducing blood pressure [15]. MMPs can also cleave vasoactive substances to produce vasoconstrictors [16-18].

In the present study, fluorospectrophotometry and gelatin gel zymography were employed to determine the plasma levels of selected MMPs of WKY and SHR. Fluorescently quenched substrates specific for MMP-1, -1/-9, $-2,-3,-7,-8,-13$, and -14 were utilized and their cleavage into fluorescent products was measured in plasma. We determined the level of VEGFR-2 proteolytic cleavage as well as its association with endothelial apoptosis and capillary rarefaction before and after chronic MMP inhibition. Among hundreds of MMP-inhibitory compounds that have been developed [19], we used doxycycline, a member of the tetracycline family, which is the only FDAapproved MMP inhibitor, and usable in future clinical studies.

\section{Materials and Methods}

\section{Reagents}

Representatives of the five subgroups of the MMP family were studied in this report. The following fluorogenic substrates were purchased (from American Peptide Company, APC, Sunnyvale, Calif., and Sigma-Aldrich, St. Louis, Mo., USA):
Vascular Collagenase: MMP-1 (collagenase, or murine collagenase-like A and B in the rat), Dnp-Pro-Cha-Abu-Cys(Me)-HisAla-Lys(N-Me-Abz)-NH, $1.5 \mu \mathrm{M}$, excitation (EX) at $365 \mathrm{~nm}$ and emission (EM) at $450 \mathrm{~nm}$ (APC); MMP-8 (neutrophil collagenase) Dnp-Pro-Leu-Ala-Tyr-Trp-Ala-Arg, $1.5 \mu \mathrm{M}$, EX/EM 280/360 nm (APC), and MMP-13 (collagenase-3) Mca-Pro-Cha-Gly-Nva-HisAla-Dpa- $\mathrm{NH}_{2}, 1.5 \mu \mathrm{M}$, EX/EM 280/360 nm (APC).

Gelatinase: MMP-2 (gelatinase A), Mca-Pro-Leu-Ala-NvaDpa-Ala-Arg- $\mathrm{NH}_{2}, 1.5 \mu \mathrm{M}, \mathrm{EX} / \mathrm{EM}$ 350/385 nm (Sigma), and MMP-1/-9 (for collagenase and gelatinase B), N-(2,4-dinitrophenyl)-Pro-Leu-Gly-Leu-Trp-Ala-D-Arg, $1.5 \mu \mathrm{M}$ EX/EM 280/346 nm (Sigma).

Stromelysin: MMP-3 (stromelysin-1), MOCAc-Arg-Pro-LysPro-Tyr-Ala-Nva-Trp-Met-Lys-(DNP)- $\mathrm{NH}_{2}, \quad 1.5 \mu \mathrm{M}, \quad$ EX/EM 325/395 nm (Sigma).

Membrane-Type MMP. MMP-14 (MT1-MMP), MCA-PLA$\mathrm{C}(\mathrm{OMeBz})-W A R(D p a)-\mathrm{NH}_{2}, \quad 1.5 \mu \mathrm{M}, \quad$ EX/EM 380/460 nm (APC).

Matrilysin: MMP-7 (matrilysin-1), Dnp-Arg-Pro-Leu-AlaLeu-Trp-Arg-Ser, $1.5 \mu \mathrm{M}$, EX/EM 278/358 nm, APC.

The specificity of the fluorogenic substrates was validated with the purified enzyme MMP-1, -2, -7, -8, -9, and -13 (Calbiochem, Gibbstown, N.J., USA). Pro-MMPs were activated with 2aminophenylmercuric acetate. VEGFR-2 was labeled with antibodies against the extracellular domain (Q-20, Santa Cruz Biotechnology, Santa Cruz, Calif., USA; binding to a 20 -amino acid epitope between amino acid sites 50 and 100) and the intracellular domain (S-20, Santa Cruz; binding to a 20-amino acid epitope between amino acid sites 1,300 and 1,350). Apoptosis was detected with TACS $\cdot \mathrm{XL}^{\circledR} \mathrm{DAB}$ in situ apoptosis.

Detection Kit (Trevigen 4828-30-DK). To block MMP activity, we used the broad-spectrum MMP inhibitors doxycycline (WestWard, Eatontown, N.J., USA), GM6001 (Calbiochem) and the iron chelator ethylene-diamine-tetraacetic acid (EDTA, Fisher Scientific, Schwerte, Germany).

\section{Animals}

The experimental protocol was reviewed and approved by the University of California San Diego Animal Subjects Committee. After general anesthesia (sodium pentobarbital, $50 \mathrm{mg} / \mathrm{kg}$ body weight i.m.; Abbott Laboratories, North Chicago, Ill., USA), male SHRs, normotensive WKY and Wistar rats (Charles River Laboratories, Wilmington, Mass., USA; 12-18 weeks, 280-350 g) were cannulated with a femoral artery and a femoral vein catheter (polyethylene catheters, PE50, Becton Dickinson Primary Care Diagnostics, Sparks, Md., USA). The mean arterial pressure and heart rate were digitally recorded (MacLab with Power Macintosh G3). Supplemental doses of anesthesia ( $5 \mathrm{mg} / \mathrm{kg}$ body weight i.v.) were administered as needed after reflex testing. The body temperature was maintained at $37^{\circ} \mathrm{C}$ by a water-heated stage. At the end of the study, the animals were euthanized (sodium pentobarbital $120 \mathrm{mg} / \mathrm{kg}$ body weight i.v.).

Subgroups of the SHRs and WKY rats were treated with the broad-blocking MMP inhibitor doxycycline $(5.4 \mathrm{mg} / \mathrm{kg} / \mathrm{day}, 24$ weeks; West-Ward) as described [15].

Plasma Protease Activities

Fresh plasma samples were collected and frozen $\left(-80^{\circ} \mathrm{C}\right)$ until assayed. For analysis, the samples were unfrozen and tested simultaneously for overall protease activity (Enzchek BODIPY, ca- 
sein derivative, catalogue No. E-6638; Molecular Probes, Carlsbad, Calif., USA; cleaved by metallo-, serine, acid, and sulfhydryl proteases) and specific protease activity with each of the intramolecularly quenched fluorescent substrates. Protease activity levels were determined from the fluorescent intensity after peptide cleavage after a 1 -hour incubation at $37^{\circ} \mathrm{C}$ (SpectraMax Gemini XS, Molecular Devices, Sunnyvale, Calif., USA; in fluorescent units). As controls, individual MMP activity levels were also determined with the fluorogenic substrates and standards of purified MMP in different concentrations $(0,1,10$, and 100 $\mathrm{nM})$.

\section{Gel Zymography Protocol}

We confirmed selected MMP activity by molecular weight determination using gelatin and carboxymethylated transferrin zymography (protocol from Bio-Rad Laboratories, Hercules, Calif., USA). Briefly, SDS gels (10\% degassed acrylamide/bis) with gelatin $(0.8 \mathrm{mg} / \mathrm{ml})$ were loaded with plasma samples and run $(\sim 125 \mathrm{~V}$, constant voltage) until the bromophenol blue tracking dye reaches the bottom of the gel. The gels were incubated in the renaturing buffer during gentle agitation for $60 \mathrm{~min}$ at room temperature. Subsequently, the gels were incubated in the developing buffer, equilibrated for $30 \mathrm{~min}$ at room temperature, and then incubated in the fresh developing buffer $\left(37^{\circ} \mathrm{C}\right)$ overnight for maximum sensitivity. MMP-7s were analyzed by $12.5 \%$ carboxymethylated transferrin zymography. Heparin was used to enhance the zymographic assays [20].

The gels were stained with Coomassie blue R-250 (30 min) and then de-stained with destaining solution (methanol:acetic acid: water, 50:10:40) until areas of gelatinolytic activity appeared as clear sharp bands (where the protease had digested the gelatin) over the blue background.

\section{VEGFR-2 Cleavage}

To examine the possibility that proteases in plasma of the SHR may cleave the extracellular domain of VEGFR-2, freshly frozen cardiac tissue sections from Wistar rats were un-frozen, then exposed for $6 \mathrm{~h}$ to plasma from the SHR, WKY, and control Wistar rats, and selected purified MMPs. After fixation in paraformaldehyde and permeabilization with saponin, the tissue sections were labeled with a primary antibody against the extracellular and separately against the intracellular domain of VEGFR-2, followed by biotinylated secondary antibody and avidin:biotinylated enzyme complex and Vector NovaRED peroxidase substrate [15]. Measurements of receptor density were determined by light intensity measurements.

\section{Cell Apoptosis Levels}

Cardiac microvessels and thymus were examined by TdT-mediated dUTP-biotin nick end labeling [terminal deoxynucleotidyl transferase-mediated dUTP nick end-labeling (TUNEL)] according to instructions (VasoTACS; Trevigen, Gaithersburg, Md., USA).

\section{Microvessel Length Density}

The vessels in cardiac muscle were immunolabeled with antibody against the intracellular domain of VEGFR-2. Stereology was used to analyze vessel density (capillary length per tissue volume) [3]. The cremaster muscle was excised and labeled with fluorescein isothiocyanate-conjugated Bandeiraea simplicifolia-1

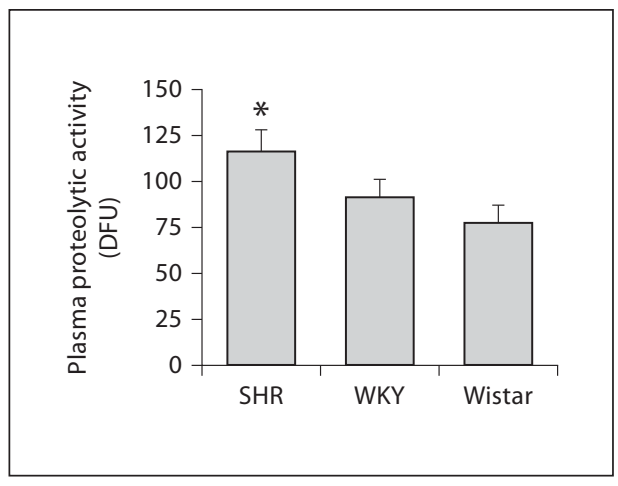

Fig. 1. Plasma protease activity (digital fluorescence units, DFU) in the SHR, WKY, and Wistar plasma ( $n=6$ /group) determined by fluorescently quenched casein. Measurements are derived from 6 rat plasma samples in each group. ${ }^{*} \mathrm{p}<0.05$.

lectin dissolved in phosphate-buffered saline (20 $\mu \mathrm{g} / \mathrm{ml}$, Sigma), which selectively bonded to small vessels with a diameter $20 \mu \mathrm{m}$. Using a confocal microscope (Bio-Rad MRC-1024UV), we obtained stacked fluorescent images and examined microvessel length density, i.e. the total vessel length per unit tissue volume, as reported previously $[3,18]$.

\section{Statistics}

All measurements are presented as means \pm SD. Comparisons of mean values between animal groups were carried out by ANOVA, and Student-Newman-Keuls test was used for multiple comparisons. Student's t test was used to determine differences between groups. $\mathrm{p}<0.05$ was considered statistically significant.

\section{Results}

\section{Arterial Blood Pressure}

The mean femoral arterial blood pressures in the WKY rats and SHRs were $113 \pm 4$ and $164 \pm 6 \mathrm{~mm} \mathrm{Hg}$, respectively $(\mathrm{p}<0.05)$.

\section{Plasma Protease Activity}

Compared to WKY and Wistar plasma, average total protease activity detected by casein in the SHR plasma was significantly increased on average by $35 \%$ (fig. 1). In addition, the SHR had a significantly elevated plasma activity of MMP-2, MMP-9, and MMP-7 (by 10.9, 25.5, and $15.0 \%$, respectively; $\mathrm{p}<0.05$ in each case; fig. 2 ). The plasma MMP-1, -8, and -13 activities in the SHR were not significantly increased $(\mathrm{p}>0.05)$. The plasma protease activity of MMP-3 was not elevated in the SHR compared to the WKY rats. In contrast, in the SHR plasma 


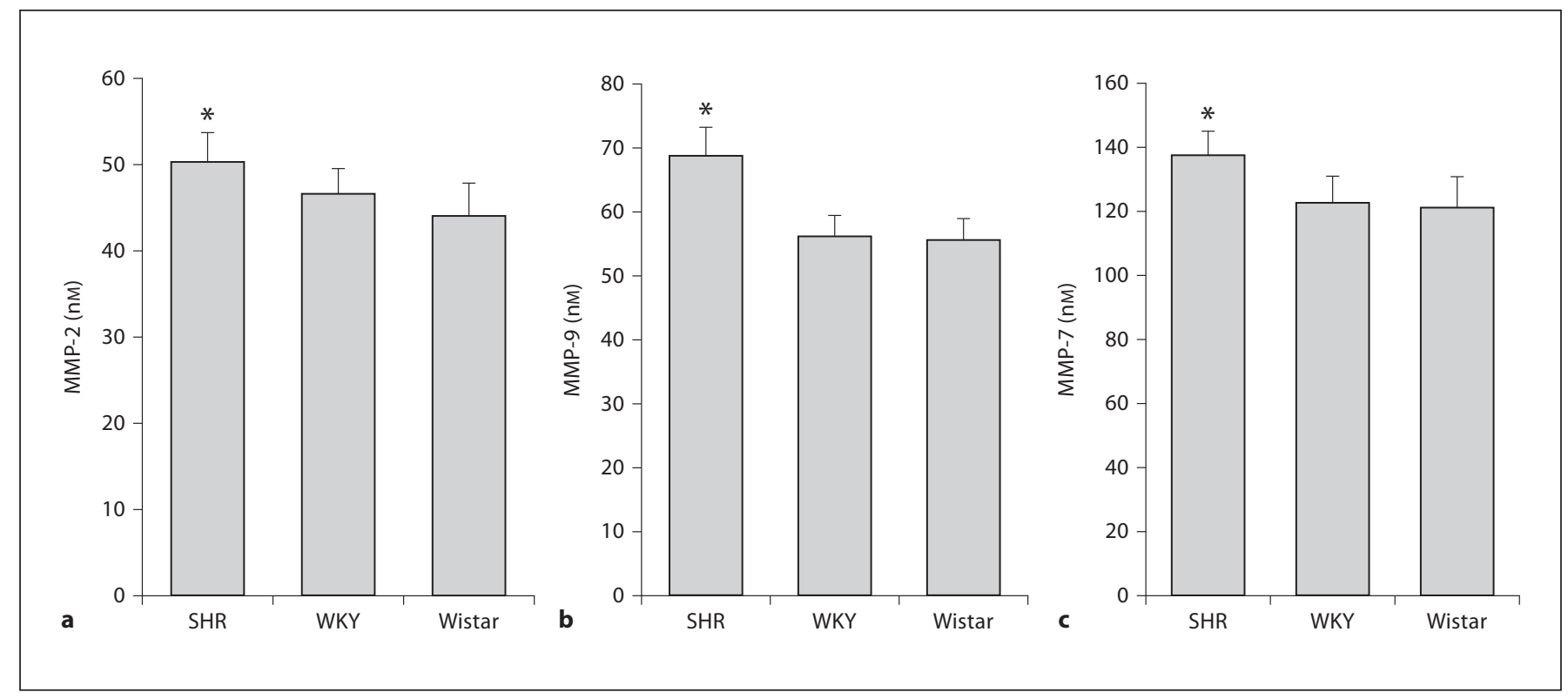

Fig. 2. Plasma MMP activity in the SHR, WKY, and Wistar plasma determined by specific fluorescently quenched substrates. Specific individual MMP equivalent activity levels were determined with the fluorogenic substrates and standards of purified
MMPs. $\mathrm{n}=6$ rat plasma samples/group. a MMP-2 equivalent units. b MMP-9 equivalent units. c MMP-7 equivalent units. ${ }^{*} \mathrm{p}<0.05$.

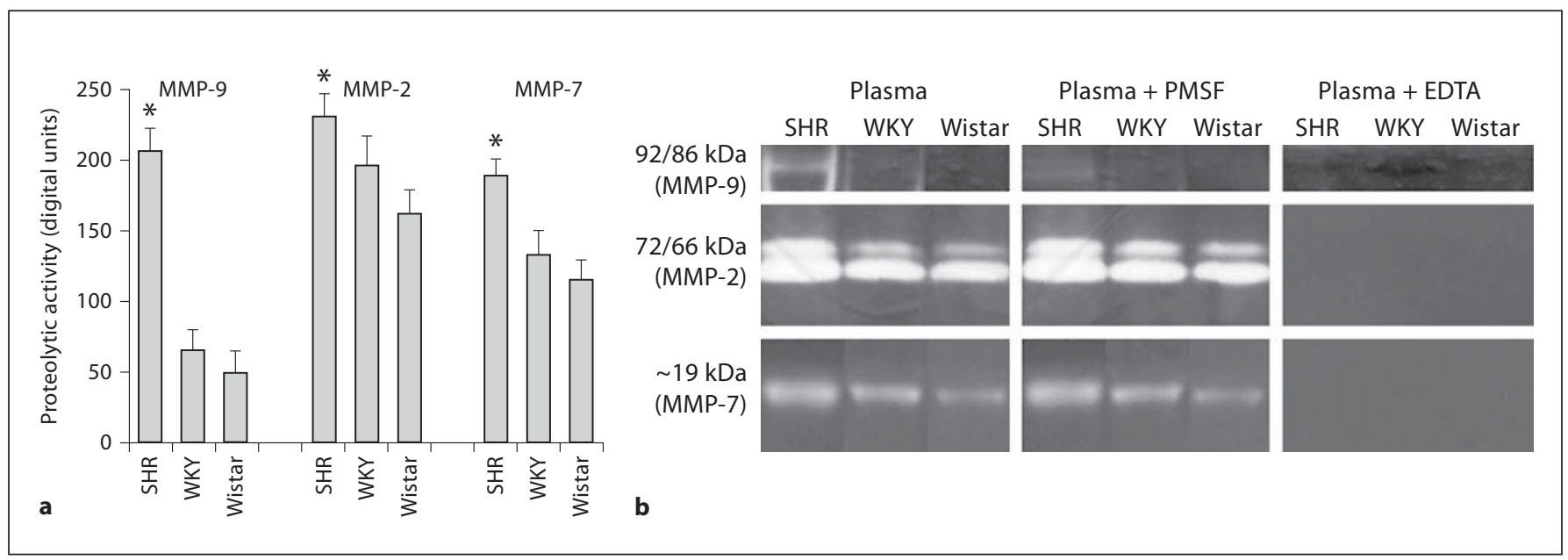

Fig. 3. Activity of specific MMPs in the SHR plasma in gelatin zymography. The protease activity in plasma was confirmed by gel zymography using molecular weight standards for confirmation. Enhanced pro- and active-MMP-9 (92/86 kDa), MMP-2 $(72 / 66 \mathrm{kDa})$, as well as MMP-7 $(\sim 20 \mathrm{kDa})$ activities were detected in the SHR plasma (b). The bar graphs (a) represent measure- ments of active MMP levels. As control, we showed that all MMP activity is blocked in vitro by metal chelation (EDTA) and we confirmed by gel zymography with a serine protease inhibitor, phenylmethylsulfonyl fluoride (PMSF), that the enhanced proteolytic activity in the SHR was not affected by serine protease inhibition (b). 
MMP-14 activity was on average $21 \%$ lower than in WKY rats $(\mathrm{p}<0.05)$.

In an alternative approach, the elevated MMP-2, MMP-9, and MMP-7 activities in SHR plasma were confirmed by gel zymography (fig. 3). As control, we showed that all MMP activity is blocked in vitro by metal chelation (EDTA; fig. 3) and by GM6001 (results not shown). We further confirmed by gel zymography with a serine protease inhibitor, phenylmethylsulfonyl fluoride, that the enhanced proteolytic activity in the SHR was not affected by serine protease inhibition.

\section{Receptor Cleavage of VEGFR-2 in the SHR}

Cleavage of the extracellular domain of VEGFR-2 was detected by use of separate antibodies against their intraand extracellular domains in cardiac tissue of the SHR and WKY. The SHR had a significantly lower expression of the extracellular domain of VEGFR-2 on endothelium of cardiac arteries, arterioles and capillaries, with a similar trend in cardiac venules (fig. 4).

\section{Receptor Cleavage of VEGFR-2 by SHR Plasma}

Application of plasma from the SHR and WKY or selected purified MMPs to naïve cardiac tissues from a normotensive Wistar rat serves to examine their ability to cleave VEGFR-2. The cleavage of VEGFR-2 was detected by use of the primary antibody against its extracellular domain. The label density of the extracellular domain of VEGFR-2 on the surface of cardiac endothelial cells was reduced by SHR plasma, as well as by MMP-7 and MMP9 (fig. 5a).

The receptor cleavage by SHR plasma was blocked by addition of MMP inhibitors, GM6001 $(1 \mu \mathrm{M})$, EDTA (10 $\mathrm{mM})$, and doxycycline $(11.3 \mu \mathrm{M} ; \mathrm{p}<0.05$ without vs. with each blocker, results not shown).

\section{Chronic MMP Inhibition Attenuated VEGFR-2}

Cleavage and Apoptosis in the SHR

Chronic doxycycline treatment attenuated the level of receptor cleavage of VEGFR-2 in cardiac tissue (fig. 5b), as well as the level of apoptosis and capillary rarefaction in the SHR heart muscle (fig. 6a, b) and also in cremaster muscle (fig. 6c). Enhanced levels of apoptosis and tissue rarefaction in the SHR thymus [21] were also significantly attenuated by chronic doxycycline treatment (results not shown). The attenuation of apoptosis and microvascular rarefaction is accompanied by a significant reduction in the SHR blood pressure [15].

\section{Discussion}

The current observations of elevated plasma levels of MMP-2, -9, and -7 activity in the SHR are in agreement with previous studies demonstrating enhanced protein expression of MMP-2, -9 , and -7 in models of hypertension, stroke, ischemia/reperfusion, and target organ injury. The SHR has elevated MMP-9 mRNA [22] compared to the WKY strain. The MMP-9 level is also elevated in stroke [23]. Levels of renal cortical MMP-9, and medullary MMP-9 and MMP-7 are increased in the SHR [24]. MMP-2 and -9 activities also contribute to glomerular injury and hypertensive remodeling [24] and to degradation of the blood-brain barrier during occlusion with reperfusion [25]. MMP-9 is observed consistently in endothelial cells and leukocytes under ischemic conditions $[26,27]$. Aging and the development/progression of hypertension are associated with increased MMP-2 activity in the aorta of the SHR [28].

MMP-7 and -9 may cleave VEGFR-2 at multiple positions (e.g. Leu-Ser|Met-Leu, Leu-Ser|Ile-Arg) [29]. We show here that the plasma MMP activity causes cleavage of the extracellular, but not intracellular, domain of VEGFR-2 on endothelium both under steady-state conditions and also when naïve Wistar cells are exposed to SHR plasma. The cleavage can also be achieved by purified MMP-7 and -9 (fig. 5a). This cleavage reduces the ability of the cell to bind VEGF agonists and may be one of the reasons for the enhanced apoptosis in the SHR endothelium [4, 30-32]. Chronic MMP inhibition with doxycycline attenuates VEGFR-2 cleavage as well as endothelial apoptosis and consequent capillary rarefaction. Further research is required to identify the exact cleavage sites and determine the fate of the cleaved fragments of the receptor.

The cleavage of the extracellular domain of VEGFR-2 in the SHR is in line with the cleavage of the extracellular domain of the insulin receptor $\alpha$ and the leukocyte adhesion receptor CD18 [15]. Receptor cleavage compromises the ability of insulin to stimulate glucose transport and leukocyte adhesion, i.e. they cause type II diabetes and immune suppression encountered in the SHR. Thus, evidence supports the idea that receptor cleavage by MMPs may compromise a range of receptor functions, i.e. cleavage of VEGFR-2 leads to endothelial apoptosis and microvascular rarefaction, cleavage of the insulin receptor causes insulin resistance, and cleavage of CD18 suppressed leukocyte adhesion to the endothelium. Chronic treatment of the SHR with an MMP inhibitor restores normal cell function and attenuates this pathophysiological process [15]. 

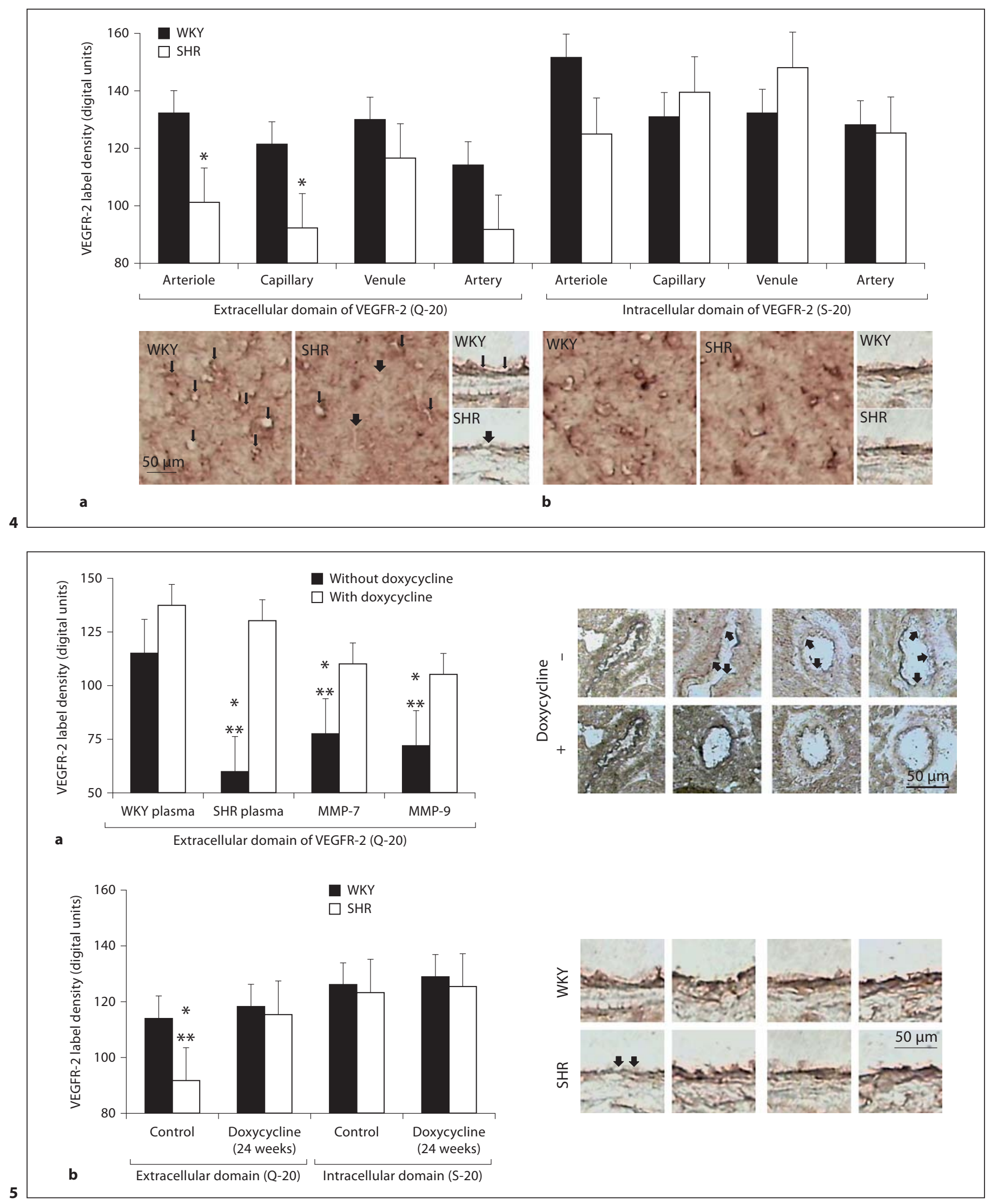

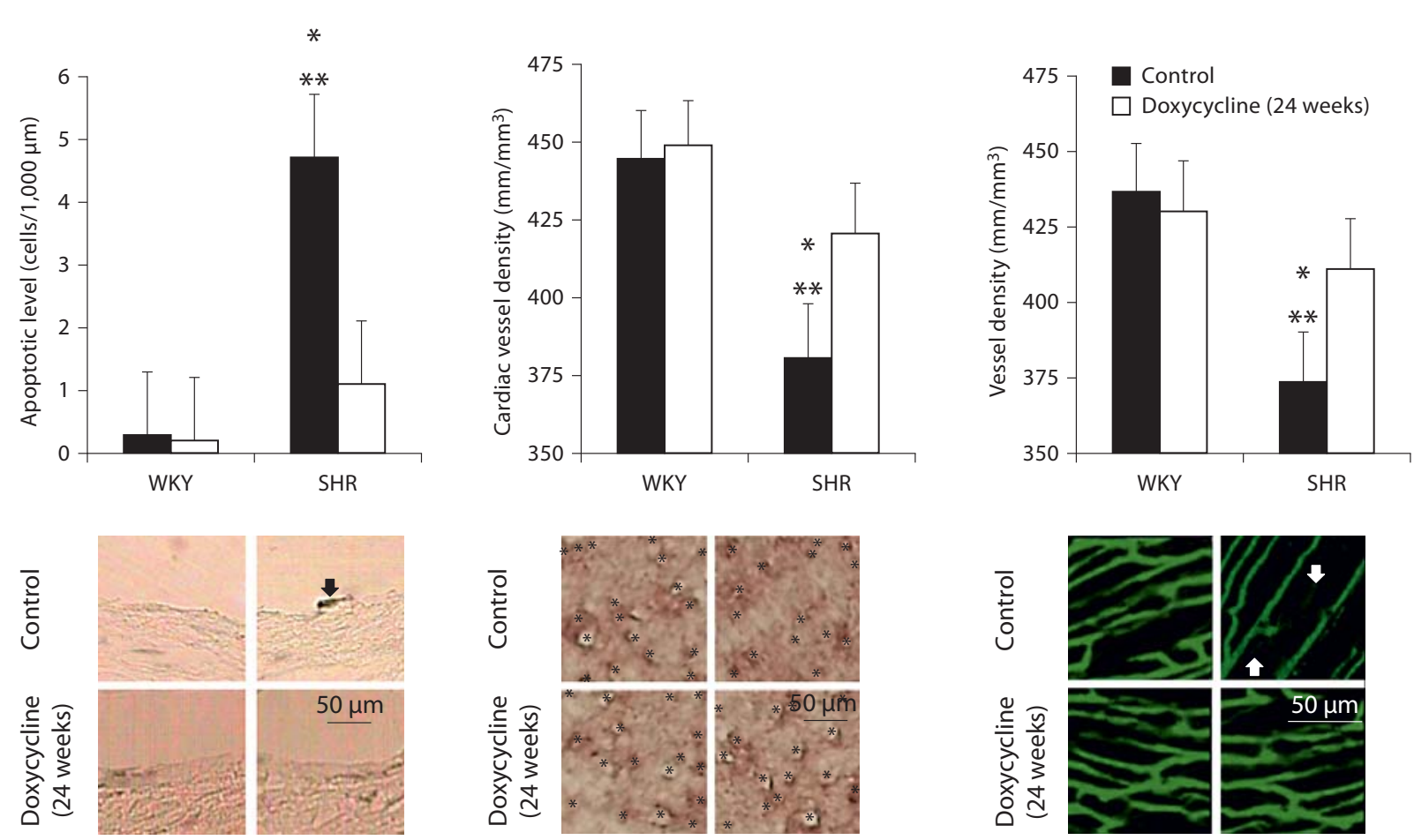

b

Fig. 4. Extracellular (a), but not intracellular (b), domain cleavage of VEGFR-2 in SHR vascular endothelium. Immunohistochemical receptor density in the rat heart microvasculature and cardiac artery were measured after labeling with separate antibodies against the extracellular (a) and intracellular (b) domain of VEGFR-2 in WKY and SHR. The SHR has a lower expression of extracellular domains of VEGFR-2 in cardiac artery, arterioles, and capillaries, with a similar trend in cardiac venules (heavy arrows point to locations with lower VEGFR-2 extracellular label, as compared to locations with the normal label density shown by small arrows). $\mathrm{n}=6$ rats/group, and 8 measurements in each vessel type; 8 arteries/heart. ${ }^{*} \mathrm{p}<0.05$ WKY vs. SHR.

Fig. 5. a Extracellular domain cleavage of VEGFR-2 by SHR plasma, MMP-7 and MMP-9. VEGFR-2 immunolabel density was measured after acute treatment (for $6 \mathrm{~h}$ ) of Wistar heart muscle sections with plasma from WKY and SHR, and with purified MMP -7 and MMP-9. There is extensive cleavage of the extracellular domain of VEGFR-2 on naïve endothelial cells (arrows). All receptor cleavage by SHR plasma, MMP-7, and MMP-9 was blocked by addition of doxycycline $(11.3 \mu \mathrm{M}$, see with doxycycline group) as well as GM6001 (1 $\mu \mathrm{M})$, EDTA (10 mM; not shown). Measurements are derived from 4 repeats per plasma/MMP group and 8 arterioles per Wistar cardiac section. ${ }^{*} \mathrm{p}<0.05$ between SHR plasma, MMPs and WKY plasma; ${ }^{* *} \mathrm{p}<0.05$ compared to case with doxycycline treatment. $\mathbf{b}$ Reversal of VEGFR-2 cleavage in the SHR by chronic MMP inhibition. VEGFR-2 label density was measured in the SHR and WKY without (control groups) and with chronic MMP inhibition (doxycycline, $5.4 \mathrm{mg} / \mathrm{kg} / \mathrm{day}, 24$ weeks; doxycycline group). The SHR exhibits many sites of sig- nificantly reduced VEGFR-2 labeling (on average about 19\%) on the extracellular domain (left images, arrows). There are no differences in VEGFR-2 expression and receptor cleavage in cardiac arterial endothelium detected between WKY rats and SHRs after treatment with the MMP inhibitor. Measurements are derived from 4 rats/group and 8 cardiac artery cross-sections/heart. ${ }^{*} \mathrm{p}<$ 0.05 comparison of the extracellular domain of VEGFR-2 density between SHR and WKY; ${ }^{* *} \mathrm{p}<0.05$ comparison of the extracellular domain of VEGFR-2 density between SHR and SHR + doxycycline.

Fig. 6. Reversal of endothelial apoptosis and capillary rarefaction by chronic MMP inhibition. TUNEL labeling of apoptotic endothelial cells, capillaries with intracellular domain of VEGFR-2 labeling in the microcirculation of the rat heart, and capillary networks in the cremaster muscle microcirculation with fluorescent lectin labeling. a The number of TUNEL-positive endothelial cells is significantly higher in SHR cardiac arteries. After chronic MMP inhibition (doxycycline, $5.4 \mathrm{mg} / \mathrm{kg} /$ day, 24 weeks), there were no significant differences in TUNEL-positive cells detected between WKY rats and SHRs. b, c The SHR has lower capillary length density ( ${ }^{*}$ for capillaries in cardiac muscle in $\mathbf{b}$, and green network in cremaster muscle in c). Chronic MMP inhibition increased microvascular length density in the SHR cremaster muscle. Apoptosis and cardiac capillary density measurements are derived from 4 rats/group and 8 cardiac cross-sections per heart. Cremaster muscle capillary density measurements are derived from 4 rats per group with one capillary region per cremaster muscle. ${ }^{*} \mathrm{p}<0.05$ SHR vs. WKY; ${ }^{* *} \mathrm{p}<0.05$ with vs. without doxycycline. 
Apoptosis and capillary rarefaction have been observed in different tissues in hypertension $[4,6,33,34]$. Compared to WKY rats, the SHR has enhanced levels of cell death in the heart and also in the thymus [34], and extensive capillary rarefaction in cremaster muscle (fig. 6). Chronic MMP inhibition with doxycycline attenuates the level of cell death and vascular rarefaction in the SHR. This evidence is in line with the observation that chronic doxycycline treatment prevents tissue destruction and apoptosis in the SHR, and enhances cell survival and improves cardiac function [35-37].

In the current experiments, doxycycline was shown to serve as an agent that blocks MMPs and receptor cleavage. As discussed previously [2], doxycycline blocks MMP-1, $-2,-8$, and -9 but also has other actions, including affinity to bivalent ions, antimicrobial activity, and serpinolytic activity. Even though several of the current results are supported by the use of alternative and more specific MMP inhibitors, there is a need for chronic MMP inhibition in the SHR with more specific inhibitors.

A question may be raised whether the MMP activity in the SHR is due to pressure elevation or due to a genetic effect. Direct immunolabeling of whole-mount speci- mens and of circulating cells that pass on a periodic basis through the higher-pressure regions of the SHR circulation (arteries and arterioles) and regions with the same blood pressure as the normotensive controls (capillaries and venules) exhibit elevated MMP levels. Thus, the elevated MMPs are not unique to those parts of the SHR circulation with elevated blood pressure. There is evidence for significant polymorphisms in the SHR [38], but their impact on MMP expression is currently undefined [39]. The capillary rarefaction is encountered in a region of the microcirculation that exhibits no significant elevation of the blood pressure in the SHR. The evidence then supports the notion that elevated MMP activity and its consequences may be not entirely caused by blood pressure elevation but may be the consequence of a genetically derived systemic mechanism.

In conclusion, the current evidence suggests that in the SHR model of hypertension capillary rarefaction may be produced by endothelial apoptosis, which in turn is caused by extracellular VEGFR-2 cleavage via unchecked enhanced MMP activity. Chronic MMP inhibition serves to attenuate the VEGFR-2 cleavage and prevent capillary rarefaction.

\section{References}

1 Arndt H, Smith CW, Granger DN: Leukocyte-endothelial cell adhesion in spontaneously hypertensive and normotensive rats. Hypertension 1993;21:667-673.

$\checkmark 2$ DeLano FA, Balete R, Schmid-Schönbein GW: Control of oxidative stress in microcirculation of spontaneously hypertensive rats. Am J Physiol Heart Circ Physiol 2005;288: H805-H812.

3 Kobayashi N, DeLano FA, Schmid-Schönbein GW: Oxidative stress promotes endothelial cell apoptosis and loss of microvessels in the spontaneously hypertensive rats. Arterioscler Thromb Vasc Biol 2005;25:21142121.

4 Lim HH, DeLano FA, Schmid-Schönbein GW: Life and death cell labeling in the microcirculation of the spontaneously hypertensive rat. J Vasc Res 2001;38:228-236.

5 Suematsu M, Suzuki H, DeLano FA, SchmidSchönbein GW: The inflammatory aspect of the microcirculation in hypertension: oxidative stress, leukocytes/endothelial interaction, apoptosis. Microcirculation 2002;9: 259-276.

6 Tran ED, Schmid-Schönbein GW: An invivo analysis of capillary stasis and endothelial apoptosis in a model of hypertension. Microcirculation 2007;14:793-804.
7 Baffert F, Le T, Sennino B, Thurston G, Kuo CJ, Hu-Lowe D, McDonald DM: Cellular changes in normal blood capillaries undergoing regression after inhibition of VEGF signaling. Am J Physiol Heart Circ Physiol 2006;290:H547-H559.

-8 Gerber HP, Hillan KJ, Ryan AM, Kowalski J, Keller GA, Rangell L, Wright BD, Radtke F, Aguet M, Ferrara N: VEGF is required for growth and survival in neonatal mice. Development 1999;126:1149-1159.

-9 Neufeld G, Cohen T, Gengrinovitch S, Poltorak Z: Vascular endothelial growth factor (VEGF) and its receptors. FASEB J 1999;13: 9-22.

$>10$ Meeson AP, Argilla M, Ko K, Witte L, Lang RA: VEGF deprivation-induced apoptosis is a component of programmed capillary regression. Development 1999;126:1407-1415.

$\checkmark 11$ Raffetto JD, Khalil RA: Matrix metalloproteinases and their inhibitors in vascular remodeling and vascular disease. Biochem Pharmacol 2008;75:346-359.

12 Wang H, Olszewski B, Rosebury W, Wang D, Robertson A, Keiser JA: Impaired angiogenesis in SHR is associated with decreased KDR and MT1-MMP expression. Biochem Biophys Res Commun 2004;315:363-368.
13 Galis ZS, Khatri JJ: Matrix metalloproteinases in vascular remodeling and atherogenesis: the good, the bad, and the ugly. Circ Res 2002;90:251-262.

14 Spinale FG: Matrix metalloproteinases: regulation and dysregulation in the failing heart. Circ Res 2002;90:520-530.

15 DeLano FA, Schmid-Schönbein GW: Proteinase activity and receptor cleavage: mechanism for insulin resistance in the spontaneously hypertensive rat. Hypertension 2008; 52:415-423.

16 Fernandez-Patron C, Radomski MW, Davidge ST: Vascular matrix metalloproteinase-2 cleaves big endothelin-1 yielding a novel vasoconstrictor. Circ Res 1999;85: 906-911.

-17 Martinez A, Oh HR, Unsworth EJ, Bregonzio C, Saavedra JM, Stetler-Stevenson WG, Cuttitta F: Matrix metalloproteinase-2 cleavage of adrenomedullin produces a vasoconstrictor out of a vasodilator. Biochem J 2004;383: 413-418.

-18 McQuibban GA, Gong JH, Tam EM, McCulloch CA, Clark-Lewis I, Overall CM: Inflammation dampened by gelatinase A cleavage of monocyte chemoattractant protein-3. Science 2000;289:1202-1206. 
19 Greenwald RA: Thirty-six years in the clinic without an MMP inhibitor. What hath collagenase wrought? Ann NY Acad Sci 1999; 878:413-419.

20 Yu WH, Woessner JF Jr: Heparin-enhanced zymographic detection of matrilysin and collagenases. Anal Biochem 2001;293:3842.

-21 Suzuki H, Miura S, Mori M, Akiba Y, Nagahashi S, Zweifach BW, Ishii $\mathrm{H}$, SchmidSchönbein GW: Protective role of adrenal glucocorticoids for gastric mucosa in spontaneously hypertensive rats. J Gastroenterol Hepatol 1999;14:364-371.

22 Hultstrom M, Leh S, Skogstrand T, Iversen BM: Upregulation of tissue inhibitor of metalloproteases-1 (TIMP-1) and procollagen$\mathrm{N}$-peptidase in hypertension-induced renal damage. Nephrol Dial Transplant 2008;23: 896-903.

-23 Tsuji K, Aoki T, Tejima E, Arai K, Lee SR, Atochin DN, Huang PL, Wang X, Montaner J, Lo EH: Tissue plasminogen activator promotes matrix metalloproteinase-9 upregulation after focal cerebral ischemia. Stroke 2005;36:1954-1959.

-24 Camp TM, Smiley LM, Hayden MR, Tyagi SC: Mechanism of matrix accumulation and glomerulosclerosis in spontaneously hypertensive rats. J Hypertens 2003;21:1719-1727.

25 Yang Y, Estrada EY, Thompson JF, Liu W, Rosenberg GA: Matrix metalloproteinasemediated disruption of tight junction proteins in cerebral vessels is reversed by synthetic matrix metalloproteinase inhibitor in focal ischemia in rat. J Cereb Blood Flow Metab 2007;27:697-709.
26 Romanic AM, White RF, Arleth AJ, Ohlstein EH, Barone FC: Matrix metalloproteinase expression increases after cerebral focal ischemia in rats: inhibition of matrix metalloproteinase-9 reduces infarct size. Stroke 1998;29:1020-1030.

-27 Rosenberg GA, Navratil M, Barone F, Feuerstein G: Proteolytic cascade enzymes increase in focal cerebral ischemia in rat. J Cereb Blood Flow Metab 1996;16:360-366.

28 Spiers JP, Kelso EJ, Siah WF, Edge G, Song G, McDermott BJ, Hennessy M: Alterations in vascular matrix metalloproteinase due to ageing and chronic hypertension: effects of endothelin receptor blockade. J Hypertens 2005;23:1717-1724.

29 Turk BE, Huang LL, Piro ET, Cantley LC: Determination of protease cleavage site motifs using mixture-based oriented peptide libraries. Nat Biotechnol 2001;19:661-667.

30 Jiang F, Zhang X, Kalkanis SN, Zhang Z, Yang H, Katakowski M, Hong X, Zheng X, Zhu Z, Chopp M: Combination therapy with antiangiogenic treatment and photodynamic therapy for the nude mouse bearing U87 glioblastoma. Photochem Photobiol 2008; 84:128-137.

31 Wang L, Dutta SK, Kojima T, Xu X, Khosravi-Far R, Ekker SC, Mukhopadhyay D: Neuropilin-1 modulates p53/caspases axis to promote endothelial cell survival. PLoS One 2007;2:e1161.

32 Xiao YF, Wu DD, Liu SX, Chen X, Ren LF: Effect of arsenic trioxide on vascular endothelial cell proliferation and expression of vascular endothelial growth factor receptors Flt-1 and KDR in gastric cancer in nude mice. World J Gastroenterol 2007;13:64986505.
\$3 Gobé G, Browning J, Howard T, Hogg N, Winterford C, Cross R: Apoptosis occurs in endothelial cells during hypertension-induced microvascular rarefaction. J Struct Biol 1997;118:63-72.

-34 Suzuki H, DeLano FA, Jamshidi N, Katz D, Mori M, Kosaki K, Gottlieb RA, Ishii H, Schmid-Schönbein GW: Enhanced DNA fragmentation in the thymus of spontaneously hypertensive rats. Am J Physiol 1999; 276:H2135-H2140.

- 35 Rossiter HB, Scadeng M, Tang K, Wagner PD, Breen EC: Doxycycline treatment prevents alveolar destruction in VEGF-deficient mouse lung. J Cell Biochem 2008;104: 525-535.

36 Xie X, Cao F, Sheikh AY, Li Z, Connolly AJ, Pei X, Li RK, Robbins RC, Wu JC: Genetic modification of embryonic stem cells with VEGF enhances cell survival and improves cardiac function. Cloning Stem Cells 2007;9: 549-563.

- 37 Yeh YC, Lai HC, Ting CT, Lee WL, Wang LC, Wang KY, Liu TJ: Protection by doxycycline against doxorubicin-induced oxidative stress and apoptosis in mouse testes. Biochem Pharmacol 2007;74:969-980.

38 Fries RS: Evidence for a Genetic Mechanism of the Chromogranin A and Phenylethanolamine-N-Methyltransferase Genes in the Pathogenesis of Hypertension in the Spontaneously Hypertensive Rat. University of California San Diego, 2007, $\mathrm{PhD}$ thesis.

- 39 Fries RS, Mahboubi P, Mahapatra NR, Mahata SK, Schork NJ, Schmid-Schoenbein GW, O'Connor DT: Neuroendocrine transcriptome in genetic hypertension: multiple changes in diverse adrenal physiological systems. Hypertension 2004;43:1301-1311. 